Cerebrovasc Dis 2010;29:309-310

DOI: $10.1159 / 000278698$

\section{Tako-Tsubo Syndrome: A Cardioembolic Cause of Brain Infarction}

\section{M.Z. Jabiria , M. Mazighia, b, P. Meimoun ${ }^{c}$, P. Amarenco ${ }^{a, b}$}

${ }^{a}$ Department of Neurology and Stroke Centre, Bichat University Hospital, and ' INSERM U-698 and Paris Diderot University - Paris VII, Paris, and ' Department of Cardiology, Compiegne Hospital, Compiegne, France

\section{Introduction}

Tako-tsubo means octopus trap in Japanese. Tako-tsubo syndrome (TS) is an acute cardiomyopathy, characterized by transient left-ventricular apical ballooning. TS is a new diagnostic entity, which mimics an acute myocardial infarction (MI), but is characterized by the absence of obstructive coronary artery disease [1]. It occurs mainly in postmenopausal women, and is frequently triggered by emotional stress. The precise incidence of TS is unknown, but up to $2.5 \%$ of all patients presenting with an initial primary diagnosis of an acute coronary syndrome (ACS) may have a TS [2-4]. This condition favors formation of an intracar- diac mural thrombus, although this seems to be an exceptional finding since thromboembolic complications occur in $0.8 \%$ of cases [2]. We report the case of a patient who had an acute brain infarction (BI) with a fully documented TS.

\section{Case Report}

A 55-year-old women, with a past medical history of cigarette smoking and alcoholism, presented with an acute chest pain in the context of a stressful situation. At the emergency department, the clinical examination was normal, but the ECG showed diffuse T-wave inversion in V3-V6-D2-D3-Vf with QT prolongation. There was a three-fold increase in troponin levels, but myoglobin was normal. Transthoracic echocardiogram (TTE) showed an extensive large apical akinetic area, an ejection fraction of $37 \%$, and no apical thrombus. Coronary angiography was normal (fig.1a, b), but ventriculography confirmed an apical ballooning (fig. 1c, d). The association of an ACS with apical ballooning at the TTE along with a normal coronary angiogram led to the diagnosis of TS. Four days later, the patient suddenly had a left-sided hemiplegia with an initial NIHSS score of 9. ECG was in sinus rhythm. DWI showed a hypersignal in the territory of the left MCA and the TOF-MRI showed a M2 segment occlusion. At $240 \mathrm{~min}$ from symptom onset, the patient had an intra-arterial rt-PA thrombolysis without any complications. At $24 \mathrm{~h}$, NIHSS score was 0
Fig. 1. Coronarography showing normal coronary arteries (a, b), and ventriculography in diastole (c) and systole (d) showing apical ballooning.
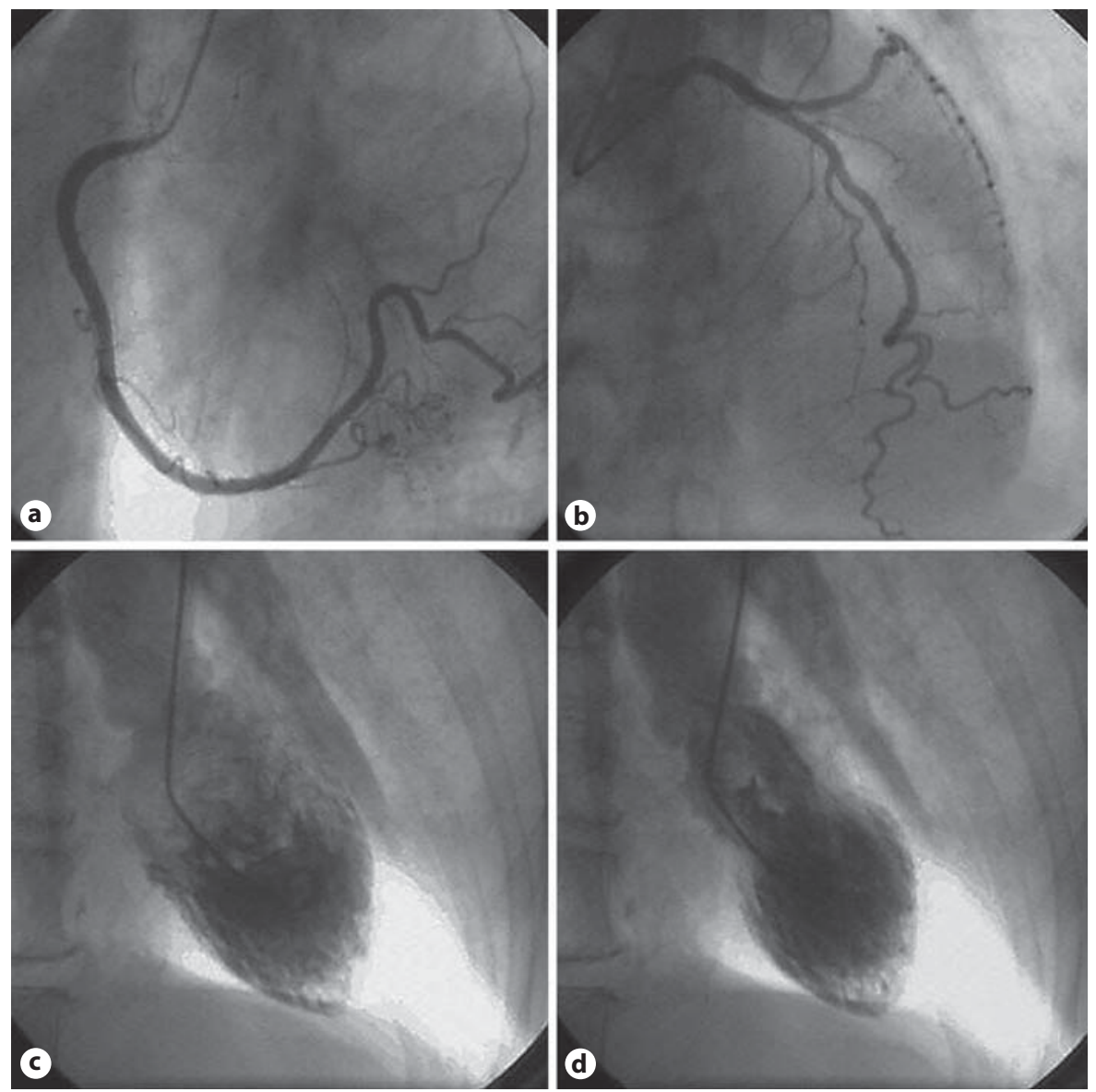
with no hemorrhagic transformation on CT scan. Carotid ultrasonography, transcranial Doppler, MRA of extracranial and intracranial vessels, and blood samples for hematological anomalies were unremarkable. Cardiac MRI performed 5 days after stroke onset was normal. Continuous cardiac monitoring and Holter ECG did not detect atrial fibrillation at any time of the course of the disease. Follow-up TTE was back to normal.

\section{Discussion}

This BI, which arose from embolic mechanisms, occurred in the course of TS. Yoshimura et al. [5] reported 7 patients with TS discovered after an BI. TS occurred between $10 \mathrm{~h}$ and 12 days after stroke. All patients were women, and 6 were aged 75 years or older. Here, TS was thought to be a consequence of subarachnoid hemorrhage or BI including the insular cortex [5]. In our case, the patient was also a postmenopausal woman, but TS preceded BI. The cardioembolic mechanism is self-evident as the wall motion abnormality of the apical region represents a condition for mural thrombus formation due to low blood flow in the apex of the left ventricle [1]. Thrombus formation in TS is not confined to the left ventricular apex. In fact, cases where the thrombus was adjacent to one of the papillary muscles have been reported [6]. Obviously, thrombus formation can be seen in any segment with a significant degree of akinesia/dyskinesia. Left ventricular thrombus formation has been reported in TS [1]. In a systematic review, de Gregorio et al. [2] found 15 TS patients with ventricular thrombosis, of which 5 had thromboembolic events, including 3 strokes.

In most patients with TS, the clinical presentation mimics an ACS. The most frequent finding on admission ECG is mild STsegment elevation typically present in the precordial leads, but ECG can be normal or show nonspecific T-wave abnormality or major ST elevation across the precordial and limb leads. TTE can detect regional wall motion abnormality, but the diagnosis is frequently made by cardiac catheterization and coronary and ventricular angiography (fig. 1). Patients with TS do not typically have coronary artery disease. Left ventriculogram shows characteristic regional ballooning involving the apical segments, but some variants of the syndrome do occur without apical involvement (mid-ventricular or reverse forms). There is sparing of the basal systolic function, and the wall motion abnormality extends beyond the distribution of a single coronary artery. Cardiac MRI may be helpful in excluding MI or differentiating from other rare causes of unobstructed coronary arteries, such as myocarditis. In the context of MI, delayed gadolinium enhancement is now the new gold standard to demonstrate wall necrosis [7], a feature neither observed in TS nor in our patient. The prognosis is generally favorable in the absence of significant underlying comorbid conditions [4]. Regional wall motion abnormalities are transient, and resolve completely within days or a few weeks $[1,5]$.

TS is an uncommon cardiomyopathy, and a potential cause of mural thrombus formation in the left ventricle. Although the prognosis of TS is usually benign, it represents a risk of embolic $\mathrm{BI}$, and stroke physicians need to maintain awareness of this fact.

\section{References}

1 Prasad A: Apical ballooning syndrome: an important differential diagnosis of acute myocardial infarction. Circulation 2007;115:e56-e59.

2 de Gregorio C, Grimaldi P, Lentini C: Left ventricular thrombus formation and cardioembolic complications in patients with takotsubo-like syndrome: a systematic review. Int J Cardiol 2008;131:18-24.

-3 Nef HM, Mollmann H, Elsässer A: Tako-tsubo cardiomyopathy (apical ballooning). Heart 2007;93:1309-1315.

-4 Gianni M, Dentali F, Grandi AM, Sumner G, Hiralal R, Lonn E: Apical ballooning syndrome or takotsubo cardiomyopathy: a systematic review. Eur Heart J 2006;27:1523-1529.

5 Yoshimura S, Toyoda K, Ohara T, Nagasawa H, Ohtani N, Kuwashiro $\mathrm{T}$, et al: Takotsubo cardiomyopathy in acute ischemic stroke. Ann Neurol 2008;64:547-554.

-6 Haghi D, Papavassiliu T, Heggemann F, Kaden JJ, Borggrefe M, Suselbeck T: Incidence and clinical significance of left ventricular thrombus in tako-tsubo cardiomyopathy assessed with echocardiography. QJM 2008; 101:381-386

$\checkmark 7$ Kim RJ, Fieno DS, Parrish TB, Harris K, Chen EL, Simonetti O, et al: Relationship of MRI delayed contrast enhancement to irreversible injury, infarct age, and contractile function. Circulation 1999;100:19922002.

Prof. Pierre Amarenco

Department of Neurology and Stroke Centre

Bichat University Hospital, 46 rue Henri Huchard

FR-75018 Paris (France)

Tel. +33 14025 8726, Fax +33 140257198

E-Mail pierre.amarenco@bch.aphp.fr 